\title{
Investigação da Pressão da Língua em Indivíduos com Miastenia Gravis
}

\author{
Investigation of Tongue Pressure in Individuals with Myasthenia Gravis
}

\author{
Marília Pinheiro de Brito Pontes ${ }^{1}$ \\ Juliana Fernandes Godoy² \\ Gabriella de França Rocha ${ }^{3}$ \\ Hébel Cavalcanti Galvão ${ }^{4}$
}

\section{RESUMO}

Objetivo: Investigar a pressão da língua em 16 pacientes com diagnóstico miastenia grave (MG). Metodologia: Foi realizado um estudo transversal para avaliar a pressão da língua em indivíduos com miastenia grave por meio do lowa Oral Performance Instrument (IOPI) e observar essa relação com a funcionalidade da deglutição. A população do estudo contemplou 16 indivíduos com miastenia grave de ambos os gêneros, na faixa etária entre 18 a 61 anos, com dentição funcional ou ajustada. Aplicou-se um questionário, registrando a presença ou ausência de queixa de deglutição, seguida de uma avaliação clínica da deglutição. Para mensuracão da pressão da língua, foi utilizado o lowa Oral Performance Instrument (IOPI) A análise dos dados foi descritiva, com nível de significância de $5 \%$ e um intervalo de confiança de $95 \%$ foi adotado para todas as análises. Resultados: A idade média dos participantes foi de $42,63 \pm 11,81 \mathrm{com}$ valores mínimo e máximo entre $18 \mathrm{e}$ 61 anos. O pico de pressão médio foi de $28.981 \pm 10,27 \mathrm{kPa}$. Nos indivíduos com disfagia, ainda que leve, o pico de pressão foi abaixo da média encontrada no grupo sem distúrbios da deglutição. Conclusão: Os pacientes com miastenia grave apresentaram perda da pressão da língua, sendo está maior naqueles que apresentam disfagia.Os achados do presente estudo, justificam a necessidade de avaliar o mecanismo de deglutição em pacientes com MG para eliminar os riscos de pneumonias aspirativas, desnutrição e desidratação ocasionado pelo fraco desempenho da musculatura da língua.

\section{DESCRITORES}

Miastenia Grave. Deglutição. Transtornos de Deglutição. Língua. Fonoaudiologia.

\begin{abstract}
Objective: Measures of tongue pressure are used to assess lingual weakness and fatigue and may have significant clinical value for the treatment of dysphagia. In the present study, the tongue pressure was investigated in 16 patients diagnosed with myasthenia gravis (MG). Methods: A cross-sectional study was carried out to assess tongue pressure in individuals with myasthenia gravis using the lowa Oral Performance Instrument (IOPI) and to observe this relationship with swallowing functionality. The study population included 16 individuals with myasthenia gravis of both genders, aged between 18 and 61 years, with functional or adjusted dentition. A questionnaire was applied, recording the presence or absence of swallowing complaints, followed by a clinical evaluation of swallowing To measure tongue pressure, the lowa Oral Performance Instrument (IOPI) was used. Data analysis was descriptive, with a significance level of $5 \%$, and a $95 \%$ confidence interval was adopted for all analyses. Results: The mean age of participants was $42.63 \pm 11.81$ with minimum and maximum values between 18 and 61 years. The mean peak pressure was $28,981 \pm 10.27 \mathrm{kPa}$. In individuals with dysphagia, although mild, the pressure peak was below the average found in the group without swallowing disorders. Conclusion: Patients with myasthenia gravis had loss of tongue pressure, which was greater in those with dysphagia. The findings of the present study justify the need to assess the swallowing mechanism in patients with MG to eliminate the risk of aspiration pneumonia, malnutrition and dehydration caused by the poor performance of the tongue muscles.
\end{abstract}

\section{DESCRIPTORS}

Myasthenia Gravis. Dysphagia. Swallowing Disorder. Tongue. Speech Terapy.

\footnotetext{
${ }^{1}$ Fonoaudióloga. Mestranda do Programa de Pós Graduação em Ciências da Saúde da Universidade Federal do Rio Grande do Norte, Macaíba, Rio Grande do Norte, Brasil.

${ }^{2}$ Fonoaudióloga. Doutora em Fonoaudiologia pela Faculdade de Bauru da Universidade de São Paulo e Professora do Departamento de Fonoaudiologia da Universidade Federal do Rio Grande do Norte, Natal, Rio Grande do Norte, Brasil.

${ }^{3}$ Fonoaudióloga, Graduada pela Universidade Federal do Rio Grande do Norte, Natal, Rio Grande do Norte, Brasil.

${ }^{4}$ Doutora em Patologia Oral pela Universidade Federal do Rio Grande do Norte e Professora Titular da Universidade Federal do Rio Grande do Norte, Natal, Rio Grande do Norte, Brasil.
} 
$\mathrm{M}$ iastenia grave autoimune (MG) é um distúrbio da junção neuromuscular, caracterizada clinicamente por fraqueza e fadiga da musculatura esquelética, com melhora após o repouso e marcada sorologicamente pela presença de autoanticorpos contra receptores de acetilcolina (AChRs), quinase específica do músculo (MuSK) ou proteína 4 relacionada à lipoproteína (LPR4) ${ }^{1}$ is $B$-cell mediated, and is associated with antibodies directed against the acetylcholine receptor, muscle-specific kinase (MUSK ${ }^{2}$.

Os dados de prevalência para MG são variáveis entre diferentes partes do mundo, entre 0,5 e 20,4 casos por 100.000 habitantes, com uma incidência anual de 0,3 por 100.000 habitantes ${ }^{3}$.

Os sintomas iniciais geralmente aparecem em um grupo muscular isolado, de preferência na musculatura ocular como ptose e diplopia. Posteriormente, os sintomas progridem com envolvimento muscular generalizado. Em 5\% dos casos, os pacientes com envolvimento bulbar referem-se como queixa inicial a disfagia, a disartria e dificuldade em mastigar e fraqueza dos músculos cervicais ${ }^{4}$.

Assim, os profissionais de saúde devem estar cientes de que as doenças neuromusculares como a miastenia grave, podem inicialmente apresentar disfagia orofaríngea como a manifestação clínica inicial da doença ${ }^{5}$.

O mecanismo normal da deglutição envolve a passagem fácil de um bolo alimentar da orofaringe para o esôfago. Os sintomas da disfagia orofaríngea geralmente surgem alguns segundos após a deglutição e os pacientes geralmente relatam escape de saliva, tosse, regurgitação nasal, aspiração ou engasgos ${ }^{6}$.
Entre os sinais de disfagia na fase orofaríngea em pacientes com MG está a diminuição da mobilidade da língua. A língua é o principal agente dessa fase. Ela forma, posiciona e faz a busca do bolo alimentar na cavidade oral. Os movimentos da língua no vestíbulo e no assoalho é uma estratégia para que nenhum resíduo alimentar permaneça na cavidade oral ${ }^{7}$. A relevância da compreensão da ação da língua em atividades como a mastigação, a deglutição e a fala ajuda a entender as disfunções e suas repercussões no complexo craniofacial $^{8}$. As disfunções na fase oral, acarretam em distúrbios na fase faríngea, que em indivíduos com MG pode repercutir no aparecimento de alterações graves como derramamento prematuro do alimento e aspiração/penetração ${ }^{9}$.

Diante desse contexto, estudos sugerem que na prática clínica, ocorra a incorporação de avaliações mensuráveis e objetivas no diagnóstico clínico dos distúrbios de deglutição, para contribuir na adaptação das estratégias terapêuticas ${ }^{10}$.

É nesse cenário, que vários estudos apresentaram dados relevantes que medem a pressão isométrica máxima que a língua exerce na cavidade oral ${ }^{11}$ associada à deglutição ${ }^{12,13}$ e em doenças neurológicas ${ }^{14,15}$. Os pesquisadores têm procurado métodos para quantificar a força da língua há muitos anos, a fim de contribuir para uma melhor intervenção nas alterações da musculatura da língua e muitos instrumentos foram construídos para esse fim. Entre as propostas de avaliação, existem relatos na literatura de um dispositivo para medir a pressão da língua, denominado lowa Oral Performance Instrument - IOPI ${ }^{16}$.

Existem evidências suficientes para apoiar o uso da IOPI como ferramenta 
adequada para medir pressão e resistência no processo de avaliação e também como ferramenta de intervenção em populações clínicas ${ }^{17}$.

$\mathrm{Na}$ literatura, faltam estudos que caracterizam alterações na deglutição como consequência de alterações na musculatura da língua causada em pacientes com MG. Assim, as altas prevalências de disfagia nas doenças neuromusculares alertam que os profissionais de saúde precisam estar atentos a esses índices e, portanto, considerar o encaminhamento desses pacientes a um fonoaudiólogo ${ }^{5}$. A obtenção de valores de pressão da língua por meio do IOPI em uma população de adultos miastênicos poderá ajudar a entender o funcionamento dessa estrutura e a diagnosticar disfunções orais que ocorrem nessa comorbidade. A compreensão dos sinais e sintomas da doença permite à equipe multiprofissional solicitar exames clínicos para avaliações focadas nos distúrbios das fases orofaríngea e esofágica, direcionando, assim, o manejo precoce da disfagia, a fim de evitar o aparecimento de morbimortalidade desses indivíduos, bem como ser capaz de desenvolver modalidades eficazes de tratamento ${ }^{18}$.

A hipótese deste estudo foi de que existe redução das medidas da pressão da língua em indivíduos com MG que pode causar distúrbios na função de deglutição. Dessa forma, o objetivo foi investigar a pressão da língua com o IOPI na elevação da língua dentro da cavidade oral.

\section{METODOLOGIA}

Estudo não randomizado transversal, aprovado pelo Comitê de Ética em Pesquisa da instituição em que foi realizado, com o Parecer $n^{\circ}$. 2.685.636. Todos os participantes assinaram o Termo de Consentimento Livre Esclarecido, após receberem as orientações a respeito do estudo. A amostra não probabilística, escolhida por conveniência, entre as pessoas que procuraram atendimento no Ambulatório de Neurologia da instituição onde aconteceu o estudo, foi composta por 16 pacientes (18 a 61 anos), dos quais 10 $(62,5 \%)$ eram do sexo feminino e $6(37,5 \%)$ do sexo masculino.

O critério de inclusão para o estudo foi ter recebido o diagnóstico de MG adquirida, fornecido pelo serviço de neurologia da instituição e estar em acompanhamento neurológico.

Coleta de dados

Foi preenchido um protocolo contendo as seguintes informações: identificação pessoal, informações sobre MG (tempo da doença, primeiros sinais e sintomas, classificação da doença, uso de medicamentos), presença ou ausência de queixa de problemas de deglutição.

Avaliação da mobilidade e pressão instrumental da língua

Em relação à avaliação da mobilidade da língua, o paciente foi solicitado a realizar protrusão de retração e estalo, com a mandíbula estável durante sua realização ${ }^{19}$. O lowa Oral Performance Instrument (IOPI), modelo 2.0, foi utilizado para medir a pressão isométrica máxima da língua. Os participantes foram aconselhados a permanecer sentados durante o procedimento, com os pés apoiados no chão e a cabeça paralela ao plano horizontal. O IOPI é formado por um transdutor de pressão que tem uma conexão com uma 
lâmpada de plástico com ar dentro dele. O dispositivo mede a pressão da língua medindo o pico de pressão máxima exercido no bulbo expresso em kilopascal ( $\mathrm{kPa}$ ). Foram realizadas três medidas para cada teste, com intervalo de descanso de 30 segundos entre elas. Foi considerada a maior medida do valor do pico pressórico dos três resultados obtidos. Para análise dos dados, considerou-se o valor médio da normalidade do pico pressórico de língua constante no manual do próprio aparelho, ou seja, $37 \mathrm{KPa}^{16}$.

Avaliação clínica da deglutição

Um protocolo clínico (Figura 1) composto por 11 perguntas e constituído por duas partes: teste de deglutição da água e teste de deglutição de alimentos pastosos, foi desenvolvido para avaliação clínica da deglutição. O protocolo foi desenvolvido por três fonoaudiólogos com experiência e extenso trabalho nas áreas de disfagia. Isso atende aos critérios apontados como fundamentais para um protocolo de aplicação clínica, uma vez que é fácil de aplicar, é rápido e abrange questões específicas sobre o que se pretende investigar. Os alimentos foram oferecidos em duas fases na avaliação funcional da deglutição: teste de deglutição com água ( $5 \mathrm{ml})$ e teste de deglutição para alimentos pastosos $(3 \mathrm{ml}, 5 \mathrm{ml}$ e $10 \mathrm{ml})$. O protocolo tem início com o teste de água, realizado por meio da oferta de $5 \mathrm{ml}$ de água, medida em seringa, mas com oferta realizada no copo. Para a oferta de dieta pastosa, o alimento foi colocado em colher de sobremesa, em porções fracionadas.

Análise estatística

Os dados foram analisados no software Statistical Package for the Social Science (SPSS) versão 20.0. Inicialmente, foi realizado o teste de Shapiro-Wilk para verificar a normalidade da amostra. A análise descritiva foi apresentada como média e desvio padrão (DP). Para avaliar a influência das variáveis na presença de disfagia, foram utilizados o teste " $\mathrm{t}$ " da amostra independente e o teste do Qui-quadrado. Em seguida, para estabelecer o limiar de força lingual, foi realizada a curva ROC (Receiver Operating Characteristic). Um nível de significância de 5\% e um intervalo de confiança de $95 \%$ foram adotados para todas as análises.

\section{RESULTADOS}

A idade média dos participantes foi de 42,63 $\pm 11,81$ anos com valores mínimo e máximo entre 18 e 61 anos. O pico de pressão da amostra estudada entre os indivíduos com disfagia leve foi significativamente $(p \leq 0,05)$ inferior à média encontrada no grupo sem distúrbios da deglutição. A caracterização da amostra e a comparação entre os grupos disfagia e não disfagia são apresentadas na Tabela 1.

De acordo com a Tabela 2, a chance de indivíduos com pressão de língua abaixo de 22,5 apresentarem disfagia leve é de $100 \%$, enquanto a chance de indivíduos com força de língua acima de 22,5 não apresentarem alteração na deglutição é de $90 \%$.

Ao realizar uma Curva Roc (Figura 1) para estabelecer qual ponto de corte tem maior chance da força de língua estar relacionada à disfagia, foi encontrado um valor de 22,5 em uma Curva Roc com área de $98,3 \%$ e valor $p=0,02$. Isso demonstra que pacientes com miastenia gravis que apresentam pressão de língua inferior a 22,5 têm maior chance de apresentar algum grau de disfagia. 
Tabela 1. Caracterização da amostra e comparação entre os grupos com disfagia e sem disfagia

\begin{tabular}{|c|c|c|c|c|}
\hline \multirow[t]{2}{*}{ Variáveis } & $\begin{array}{c}\text { Amostra } \\
n=16\end{array}$ & $\begin{array}{c}\text { Grupo com } \\
\text { disfagia leve } \\
n=6\end{array}$ & $\begin{array}{l}\text { Grupo sem } \\
\text { alteração na } \\
\text { deglutição } \\
n=10\end{array}$ & \multirow[t]{2}{*}{$\mathrm{p}$ valor } \\
\hline & \multicolumn{3}{|c|}{ Média e desvio padrão } & \\
\hline Idade & $42,63( \pm 11,81)$ & $46,33( \pm 12,95)$ & $40,40( \pm 11,19)$ & 0,34 \\
\hline Ano do diagnóstico médico & $6,81( \pm 6,13)$ & $7,00( \pm 3,57)$ & $6,70( \pm 7,45)$ & 0,95 \\
\hline Idade dos primeiros sintomas & $35,63( \pm 13,05)$ & $38,50( \pm 12,81)$ & $33,90( \pm 13,56)$ & 0,51 \\
\hline \multirow[t]{2}{*}{ Média do pico pressórico } & $28,81( \pm 10,27)$ & $19,50( \pm 2,34)$ & $34,40( \pm 8,95)$ & $0,001^{*}$ \\
\hline & \multicolumn{3}{|c|}{$n(\%)$} & \\
\hline $\begin{array}{l}\text { Sexo } \\
\text { Masculino } \\
\text { Feminino }\end{array}$ & $\begin{array}{l}06(37,5) \\
10(62,5)\end{array}$ & $\begin{array}{l}03(50) \\
03(50)\end{array}$ & $\begin{array}{l}03(30) \\
07(70)\end{array}$ & 0,60 \\
\hline $\begin{array}{l}\text { Dificuldade para comer } \\
\text { Sim } \\
\text { Não }\end{array}$ & $\begin{array}{c}12(75) \\
02(25)\end{array}$ & $06(100)$ & $\begin{array}{l}06(60) \\
04(40)\end{array}$ & 0,23 \\
\hline
\end{tabular}

Tabela 2. Associação entre o grau de disfagia e a pressão da língua

\begin{tabular}{l|ccc}
\hline \multicolumn{5}{c}{ Grau de Disfagia } \\
\hline Força da língua & Normal e Funcional & Disfagia Leve & Qui Quadrado \\
Abaixo de 22,5 & 1 & 6 & 0,001 \\
Acima de 22,5 & 9 & - & \\
\hline
\end{tabular}

Figura 1. Curva Roc para estabelecer qual ponto de corte tem maior chance de a força da língua estar relacionada à disfagia

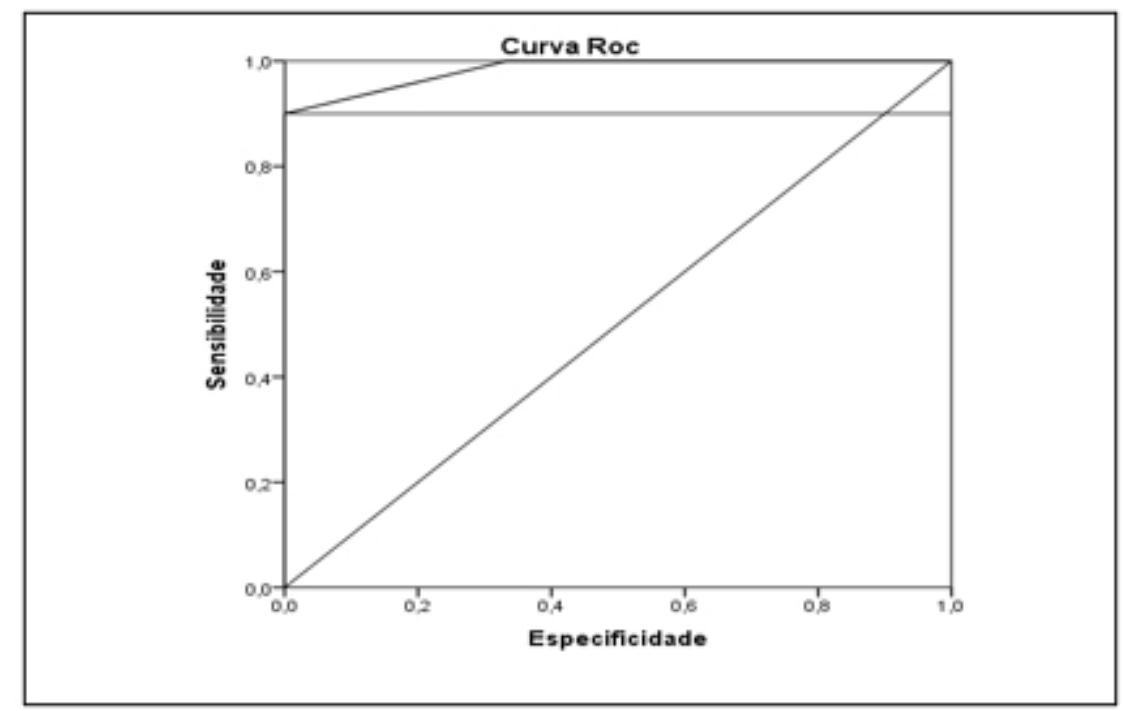




\section{DISCUSSÃO}

O interesse em investigar o pico de pressão da língua foi para identificar precocemente quadros de disfagia em indivíduos miastênicos que apresentassem média inferior no pico de pressão de língua. Além disso, era esperado que alterações na função de deglutição estivessem presentes no grupo de indivíduos estudados, o que foi confirmado pelos resultados deste estudo.

Uma especificidade dos resultados aqui encontrados permitiu analisar que nos indivíduos idosos ocorreu um maior decréscimo da pressão máxima ${ }^{20}$. Dois indivíduos maiores de 60 anos apresentaram pico pressórico inferior a 22,5, com sinais de disfagia leve e presença de engasgo. $O$ pico médio de pressão tendeu a ser menor em indivíduos miastênicos mais velhos, convergindo com estudo. Porém, embora não seja significativo, a análise dos dados permite inferir que quanto maior a idade do indivíduo com miastenia, menor o valor do pico de pressão.

O início da MG pode ocorrer em qualquer idade, mas os pacientes do sexo masculino neste estudo tiveram uma idade de início mais alta em comparação às mulheres. Assim, a idade de início dos primeiros sintomas nas mulheres foi aos 15 anos, enquanto nos homens foi aos 21 anos para. Esses dados são semelhantes aos relatados por Grob et al. ${ }^{20}$, que definem que essas incidências de $M G$ em relação à idade e sexo são semelhantes às da maioria das outras doenças autoimunes.

A maioria dos pacientes desta amostra era do sexo feminino (62,5\%). A ocorrência de MG é influenciada por sexo e idade. Estudo ${ }^{21}$ mostrou que as taxas de incidência são maiores em mulheres com faixa etária entre 35 e 44 anos, porém, apresenta taxa maior em homens mais velhos (> 65 anos).

Os primeiros relatos são frequentemente sintomas de ptose palpebral ou diplopia ${ }^{22}$. Os indivíduos miastênicos apresentam envolvimento da musculatura ocular externa em $90 \%$ a $95 \%$ dos indivíduos. Neste estudo, $31,3 \%$ dos indivíduos participantes relataram a presença de ptose palpebral ou diplopia como a primeira manifestação clínica.

A pressão máxima média (Pmax) em indivíduos de uma população normal está na faixa de 40 a $80 \mathrm{kPa}$, com média de cerca de $63 \mathrm{kPa}^{15}$. O valor médio da pressão da língua $(28,4 \pm 10,1 \mathrm{KPa})$ em pacientes com MG neste estudo ficou abaixo de outros resultados relatados na literatura para indivíduos jovens $(62,08 \pm 12,43)$, bem como em relação aos idosos $(50,75 \pm 15,25)^{7}$.

Não existe na literatura um valor estabelecido de pressão de língua para indivíduos considerados com fraqueza nesta musculatura. No entanto, essa fraqueza pode ser estimada se a Pmax for inferior a 34 $\mathrm{kPa}^{15}$. Os resultados deste estudo sugerem a hipótese de que a fatigabilidade é um fator contribuinte para problemas motores orais de pacientes com MG, uma vez que dez $(62,5 \%)$ indivíduos apresentaram pressão máxima inferior a $34 \mathrm{kPa}$. Destes, dez identificados na avaliação clínica da deglutição com deglutição normal e funcional e dois indivíduos foram classificados com disfagia orofaríngea leve.

É interessante notar que sinais de disartria e disfagia são encontrados em pacientes com doenças da junção neuromuscular, mas muitos não relatam essa queixa por apresentarem sintomas iniciais leves, o que causa uma subnotificação dos serviços de saúde para esses distúrbios ${ }^{4}$.

Nos casos de MG, que começam com 
uma síndrome bulbar, a suspeita clínica é simples, se o paciente apresentar sintomas de ptose, diplopia, disartria, disfagia e fraqueza ${ }^{3}$. Em indivíduos que apresentam a função bulbar comprometida é possível que a disfagia se apresente mais grave, com presença de penetração e aspiração laringotraqueal à medida que a doença progride ${ }^{24}$

Em um estudo ${ }^{22}$ com 58 pacientes, a disfagia apareceu como o terceiro sintoma clínico apresentado em indivíduos miastênicos. Esse achado corrobora os resultados do presente estudo, nos quais a disfagia foi observada em $12,5 \%$ dos pacientes, representando a terceira manifestação mais frequente. Este fato pode ser explicado em virtude do comprometimento da musculatura do sistema estomatognático, dos músculos da mímica facial, os mastigatórios e o orbicular dos lábios serem os mais afetados, seguidos pelos músculos da língua e véu do palatino ${ }^{21}$.

O processo de alimentação pode gerar distúrbios na fase de preparação oral, com resultados que demonstram que as funções olfativas e gustativas diminuem significativamente em pacientes com MG, o que pode gerar desinteresse pela alimentação e, assim, levar o indivíduo para perda de peso e desnutrição, de acordo com relato encontrado na literatura ${ }^{25}$.

Nessa perspectiva, a disfagia é um sintoma significativo e envolve má função dos movimentos da língua, levando à regurgitação como resultado da disfunção velofaríngea e fraqueza do peristaltismo faríngeo ${ }^{26}$.

A obtenção desses dados pode auxiliar na compreensão da evolução da doença e orienta o processo de reabilitação miofuncional. Além disso, pode contribuir para o diagnóstico de distúrbios orofaciais, contribuindo para melhorar a qualidade de vida desses indivíduos miastênicos e possibilita a perspectiva de uma abordagem multiprofissional no tratamento desses pacientes.

Este estudo tem algumas limitações. Os resultados deste estudo são um dos primeiros a oferecer uma percepção das diferenças no desempenho muscular da língua em indivíduos com MG. Porém, tratou-se de um estudo piloto com amostra relativamente pequena, por se tratar de uma coleta realizada em apenas um ambulatório de referência e os participantes serem, em sua maioria, do interior do estado e depender do transporte municipal para retornar para a cidade de origem. $O$ controle desses fatores pode reduzir ainda mais a participação no estudo. Não se sabe se o desempenho muscular da língua também seria diferente entre indivíduos com outras doenças neuromusculares.

\section{CONCLUSÃO}

As dificuldades no diagnóstico médico inicial da MG ocorrem devido aos desafios diagnósticos, como heterogeneidade na apresentação clínica e sinais e sintomas da doença que se sobrepõem a outras condições. Porém, a diminuição da língua, observada após a avaliação com o IOPI, pode estar presente no início do curso da doença e pode permanecer mesmo após a inserção do indivíduo em tratamento medicamentoso. Este estudo demonstrou que o pico de pressão da língua diminuiu moderadamente em indivíduos com MG. Na avaliação clínica da deglutição, ocorreu alteração na dinâmica da deglutição para as consistências líquida e pastosa. Esses resultados podem ajudar, no futuro, a identificar novas estratégias para melhorar o diagnóstico e a reabilitação dos distúrbios da deglutição em indivíduos com MG com perda do desempenho muscular da língua. 


\section{REFERÊNCIAS}

1. Gilhus NE, Verschuuren JJ. Myasthenia gravis: Subgroup classification and therapeutic strategies. Lancet Neurol. 2015;14(10):1023-36.

2. Aragonès JM, Altimiras $J$, Roura $P$, Alonso $F$, Bufill $E$, Munmany $A$, et al. Prevalence of myasthenia gravis in the Catalan county of Osona. Neurol. 2017; 32(1):1-5.

3. Morís G. c. Miastenia gravis y trastornos relacionados con la unión neuromuscular. Med.2019;12(76):4469-77.

4. Knuijt S, Kalf JG, De Swart BJM, Drost G, Hendricks $\mathrm{HT}$, Geurts $\mathrm{ACH}$, et al. Dysarthria and dysphagia are highly prevalent among various types of neuromuscular diseases. Disabil Rehabil. 2014; 36(15):1285-9.

5. Odufalu F. Dysphagia. Mo Med. 2018; 115(3):206-10.

6. Real CS, Balbinot J, Signorini AV, Hübner LS, Machado $\mathrm{G}$ de C, Dornelles S. Caracterização do escape posterior tardio na deglutição. CoDAS. 2020;32(4):e20190072.

7. VanRavenhorst-Bell HA, Mefferd AS, Coufal KL, Scudder $\mathrm{R}$, Patterson J. Tongue strength and endurance: Comparison in active and non-active young and older adults. Int J Speech Lang Pathol. 2017;19(1):77-86.

8. Im S, Suntrup-Krueger S, Colbow S, Sauer S, Claus I, Meuth SG, et al. Reliability and main findings of the flexible endoscopic evaluation of swallowing-Tensilon test in patients with myasthenia gravis and dysphagia. Eur J Neurol. 2018; 25(10):1235-1242.

9. Rommel N, Hamdy S. Oropharyngeal dysphagia: Manifestations and diagnosis. Nat Rev Gastroenterol Hepatol. 2016;13(1):49-59.

10. Herrera Lorenzo O, Infante Ferrer J, Casares Albernas F. Miastenia Gravis: diagnóstico y tratamiento. Rev Arch Médico Camagüey. 2009;13(5):0-0.

11. Prandini EL, Totta T, Bueno M da RS, Rosa RR, Giglio LD, Trawitzki LVV, et al. Análise da pressão da língua em indivíduos adultos jovens brasileiros. CoDAS. 2015; 27(5):478-482.

12. Park JS, You SJ, Kim JY, Yeo SG, Lee JH. Differences in orofacial muscle strength according to age and sex in East Asian healthy adults. Am J Phys Med Rehabil. 2015; 94(9):677-686

13. Mano T, Katsuno M, Banno H, Suzuki K, Suga N, Hashizume A, et al. Tongue pressure as a novel biomarker of spinal and bulbar muscular atrophy. Neurology. 2014; 82(3):255-262

14. Berggren KN, Hung M, Dixon MM, Bounsanga J, Crockett $B$, Foye MD, et al. Orofacial strength, dysarthria, and dysphagia in congenital myotonic dystrophy. Muscle and Nerve. 2018;58(3):413-417.

15. IOPI Northwest. lowa oral performance instrument: user's manual. Carnation: IOPI Northwest; 2011.
16. Vanderwegen J, Guns C, Van Nuffelen G, Elen R, De Bodt M. The influence of age, sex, bulb position, visua feedback, and the order of testing on maximum anterior and posterior tongue strength and endurance in healthy Belgian adults. Dysphagia. 2013; 28(2):159-166

17. Adams V, Mathisen B, Baines S, Lazarus C, Callister R Reliability of measurements of tongue and hand strength and endurance using the lowa Oral Performance Instrument with elderly adults. Disabil Rehabil. 2015;37(5):389-395.

18. Perilo TV de C, Motta AR, Casas EB d Las, Saffar JME, Costa CG da. Avaliação objetiva das forças axiais produzidas pela língua de crianças respiradoras orais. Rev Soc Bras Fonoaudiol. 2007;12(3):184-190.

19. Magalhães Junior, et al. Caracterização da pressão da língua em idosos. Audiol., Commun. Res. vol.19 no.4 São Paulo Oct.Dec. 2014.

20. Grob D, Brunner N, Namba T, Pagala M. Lifetime course of myasthenia gravis. Muscle and Nerve. 2008;37(2):141149.

21. Breiner A, Widdifield J, Katzberg HD, Barnett C, Bril V, Tu K. Epidemiology of myasthenia gravis in Ontario, Canada. Neuromuscul Disord. 2016;26(1):41-6.

22. Gonzalez, RL et al. Miastenia gravis: resultados inmediatos y alejados de la timectomía transesternal extendida. Rev Med Chile 2018; 146(4):460-469.

23. Mourão A, Gomez R, Barbosa L, Freitas D, Comini-Frota $E$, Kummer A, et al. Determinants of quality of life in Brazilian patients with myasthenia gravis. Clinics. 2016 71(7):370-374.

24. Brandão BC, Galdino A de S, Lourenção LG, Trindade GS, Orate MA, Silva M da, et al. Correlation between bulbar functionality and laryngeal penetration and/or laryngotracheal aspiration on motor neuron disease. Codas. 2018; 30(1):1-5.

25. Tekeli H, Senol MG, Altundag A, Yalcinkaya E, Kendirli $\mathrm{MT}$, Yaşar $\mathrm{H}$, et al. Olfactory and gustatory dysfunction in Myasthenia gravis: A study in Turkish patients. J Neurol Sci. 2015; 356(1-2):188-192.

26. Davison SP, McDonald TJ, Wolfe ME. Swollen tongue: A presentation of Myasthenia gravis. Otolaryngol - Head Neck Surg. 1997; 116(2):244-246.

\section{CORRESPONDÊNCIA}

Marília Pinheiro de Brito Pontes

Avenida Governador Juvenal Lamartine, 1012.

Tirol, Natal/RN, Brasil. Cep: 59022-020

Email:mariliapbrito@hotmail.com 